\title{
Kriging-based spatial interpolation from measurements for sound level mapping in Urban Areas
}

\author{
Pierre Aumond ${ }^{\mathrm{a}}$, Arnaud Can ${ }^{\mathrm{a}}$, Vivien Mallet ${ }^{\mathrm{b}}$, Bert De Coensel ${ }^{\mathrm{c}, \mathrm{e}}$, Carlos Ribeiro $^{\mathrm{d}}$, Dick \\ Botteldooren $^{\mathrm{e}}$, Catherine Lavandier ${ }^{\mathrm{f}}$ \\ 'Ifsttar, AME-LAE (Laboratoire d'Acoustique Environnementale), F-44341 Bouguenais, France \\ ${ }^{b}$ Inria, Paris Research Center, France \\ ${ }^{c}$ ASAsense, Belgium \\ ${ }^{d}$ Bruitparif, France \\ ${ }^{e}$ Waves Research Group, Department of Information Technology, Ghent University, Technologiepark-Zwijnaarde 15, B-9052 Ghent \\ ${ }^{f}$ ETIS, CNRS UMR8051, ENSEA, Université de Cergy-Pontoise, France
}

\section{Abstract}

Network-based noise monitoring systems are deployed in various cities over the world and mobile applications allowing participatory sensing are now common. Nevertheless, the sparseness of the collected measurements, either in space or in time, complicates the production of noise maps. This paper describes the results of a measurement campaign that has been conducted in order to test different spatial interpolation strategies for producing noise maps. Mobile measurements have been performed while walking multiple times in every street of the XIII'th district of Paris. By adaptively constructing a noise map on the basis of these measurements, the role of the density of observations and the performance of four different interpolation strategies is investigated. Ordinary universal Kriging methods are assessed, as well as the effect of using an alternative definition of the distance between observation locations, which takes the topology of the road network into account. The results show that a high density of observation points is necessary to obtain an interpolated noise map close to the reference map. Furthermore, the part of the variance explained by the alternative distance definition is small, as compared to the part of the variance explained by the addition of a simple local linear trend that is defined based on the distance between each observation location and the closest roads to that location.

Keywords: Kriging; Spatial interpolation; Sound map; Opportunistic measurements

\section{I.Introduction}

The Directive 2002/CE/49 contributed to the development and harmonization of noise prediction models (1). For making urban noise maps, model-based numerical engineering methods are currently widely used and these methods provide a good compromise between accuracy and computation time (2). Nevertheless, they have many limitations, and the resulting noise maps neglect the diversity of urban sound environments in terms of both sound sources and sound environment dynamics.

As a response, noise maps based on measurements are gaining interest (3-6). The recent development of small and autonomous acoustic sensors contributes to this movement, and network-based noise monitoring systems are being deployed in an increasing number of cities over the world, using either highquality or low-cost micropones $(4,7,8)$. Also, smartphone applications, allowing participatory sensing, are now common, which multiplies the amount of available data to potentially map the sound environment of a city based on measurements (9-12).

Nevertheless, the production of noise maps based on measurements is complicated by metrological issues inherent to the typical microphones used in consumer electronics, the time sparseness of the 
measurements collected through mobile monitoring applications, and the space sparseness of the measurements collected through fixed noise monitoring networks. Therefore, it is fundamental to know the time and space representativeness of such measurements, as this knowledge is required to be able to propose relevant interpolation methods that can be used to produce noise maps that cover the full temporal and spatial extent of the study area.

The temporal structure of urban sound levels (highly correlated day or week patterns, seasonal trends) can be exploited to restrict the number of sampled days, or to rely only on measurements performed at selected periods of the day, in order to estimate Lden values or Daily Average Noise Patterns (5,13-15). Previous studies also revealed that a 10 or 15-minute measurement is representative of a $1 \mathrm{~h}$-period in an urban context, as the majority of the 10 or 15 minute-measurements are in the same sound level range during homogeneous periods $(16,17)$. Other studies that rely on short-term recording methods proved the relevance of 15-minute sampling periods $(18,19)$. Even shorter measurement periods can be found in the literature, especially in those cases where the opportunistic measurement context offered by smartphone applications is used. In this case, the short measurement duration is compensated by the large number of measurements (10), shifting the focus from the duration of each measurement episode to the number of sampling episodes, as recommended in (20).

Zuo et al. (15) showed that the sound level variability in urban environments can be explained for a large part by the spatial characteristics of the environment. Also, the space representativeness and the spatial interpolation of the measurements is an important issue when computing sound maps based on measurements. Maps interpolated from the data obtained through fixed noise measurement stations have recently been produced $(6,21-23)$, and form a useful tool to estimate the noisiness of a neighborhood or to give a global overview of the city sound levels. However, the large distance between measurement stations does often not allow to map noise levels in each street, which is offered by maps calculated using model-based numerical methods. A study by Can et al. (24) that involved mobile measurements performed using sound level meters attached to backpacks permitted to compare an interpolated map with a reference map, but only for a very small area (four streets). More studies are therefore needed to investigate the density of measurements that is required to have an acceptable accuracy at the street resolution.

Another parameter to take into account is the method of interpolation. Several methods have been tested for urban sound level interpolation: Inverse Distance Weighting (IDW) methods $(5,6,22,24)$, Kriging methods $(6,22,24)$ and multiquadratic interpolation (6). Alternative interpolation methods that involve a modified definition of the distance between measurement locations, in order to account for the city geometry or the road network, have recently been proposed (24-26). Nevertheless, these interpolation methods have only been tested on small measurement samples, and larger studies are needed to validate the conclusions of these works. It is worth mentioning that the density of measurements appears to be more important than the method of interpolation (6).

Recently, a number of data fusion techniques have been proposed to correct model-based numerically computed noise maps with measurements $(3,26-28)$. These techniques are promising, but require a precalculated noise map, which can be expensive, and restrict the potential indicators to those that are available in this noise map. Most of the existing noise maps involve the energy-equivalent sound level, whereas in situ measurements allow to calculate a wide range of acoustical indicators, which may include information about the temporal dynamics of the sound environment; for example, percentile or emergence 
indicators can also be interesting to interpolate. The spatial interpolation methods can even be based on perceptual assessments (29).

In this study, a large measurement campaign has been conducted in the XIII ${ }^{\text {th }}$ district of Paris, with the goal to test different spatial interpolation strategies. Mobile measurements have been performed with noise measurement stations attached to backpacks, that were carried by researchers when walking in every street of the district between 1 and 15 times. The measurements are aggregated over a grid of locations in the study area, and are used to compute a reference map of the district. An analysis of the sensitivity of the sound level values with respect to the radius of the integration and the number of measurements is done via a bootstrap method. From the reference sound map, four Kriging methods for interpolation between a set of measurement locations are tested, based on a combination of two strategies: (i) Ordinary Kriging and universal Kriging which consists of adding a linear trend, defined from the distance between an observation location of the domain and its closest categorized road, and (ii) a variation of the distance definition between observation locations, which can be Euclidian or computed from the road network to take into account the influence of the city geometry. By progressively decreasing the number of observation locations, the impact of the density of observation locations and the performance of different spatial interpolation methods is investigated.

\section{II.Method}

\section{A. Study area}

Figure 1 presents the study area, which corresponds to the XIII ${ }^{\text {th }}$ District of Paris. This district includes a large variety of urban sound environments: large avenues with high traffic density, lively streets with bars and restaurants, schools, small and large parks, quiet streets. The size of the study area is approximately $2.8 \mathrm{~km}^{2}$ with a maximum extent of $2 \mathrm{~km}$ west to east and a maximum extent of $1.7 \mathrm{~km}$ north to south.

\section{B. $\quad$ Measurement set-up}

All measurements were carried out using dedicated mobile sound measurement stations developed by ASAsense (30). These stations record the instantaneous 1/3-octave band spectrum with a 125 -ms temporal resolution as well as the instantaneous GPS position with a 1-s temporal resolution. Both noise and GPS data are synchronized, such that the spatio-temporal evolution of the noise spectrum during each measurement session can be reconstructed afterwards. In order to fully capture the characteristics of the sound environment, a large set of indicators is calculated on the basis of this $1 / 3$-octave band spectrum data, including the A-weighted energy-equivalent sound level at each second, $L_{e q, I s}$, which is used throughout this study.

\section{C. $\quad$ Mobile measurements}

The sound measurement stations were mounted in backpacks with power provided by a battery pack, and, subsequently, mobile measurements were carried out between October $22^{\text {th }} 2014$ and May $26^{\text {th }} 2015$. Five operators participated in the measurements. In order to minimize the variation between measurement sessions and to be able to calculate sound levels that are representative for homogeneous sound environments, measurements were only carried out on weekdays, either between 10 a.m. and 12 a.m., or between 2 p.m. and 4 p.m. As shown in (15-17), these periods, which exclude rush hour traffic and lunch 
times, provide a similar sound environment. Depending on the variability of the sound environment, the number of walks in each street was varied. As shown in (17), the sound environment of a calm street is more sensitive to single noise events than that of a large boulevard, thus a higher number of measurements is required to calculate a sound level value that is representative of the sound environment of a calm street, as compared to a large boulevard. After each day of measurements, the variance of the sound level was computed for each street, providing feedback on those streets that would benefit from more measurements to get a stable estimate. The number of passages per street ranged from 1 to 15 times, with an average of 4 and a standard deviation of 2.7 passages. Figure 1 shows the number of walks (only validated measurements were kept to plot this figure).

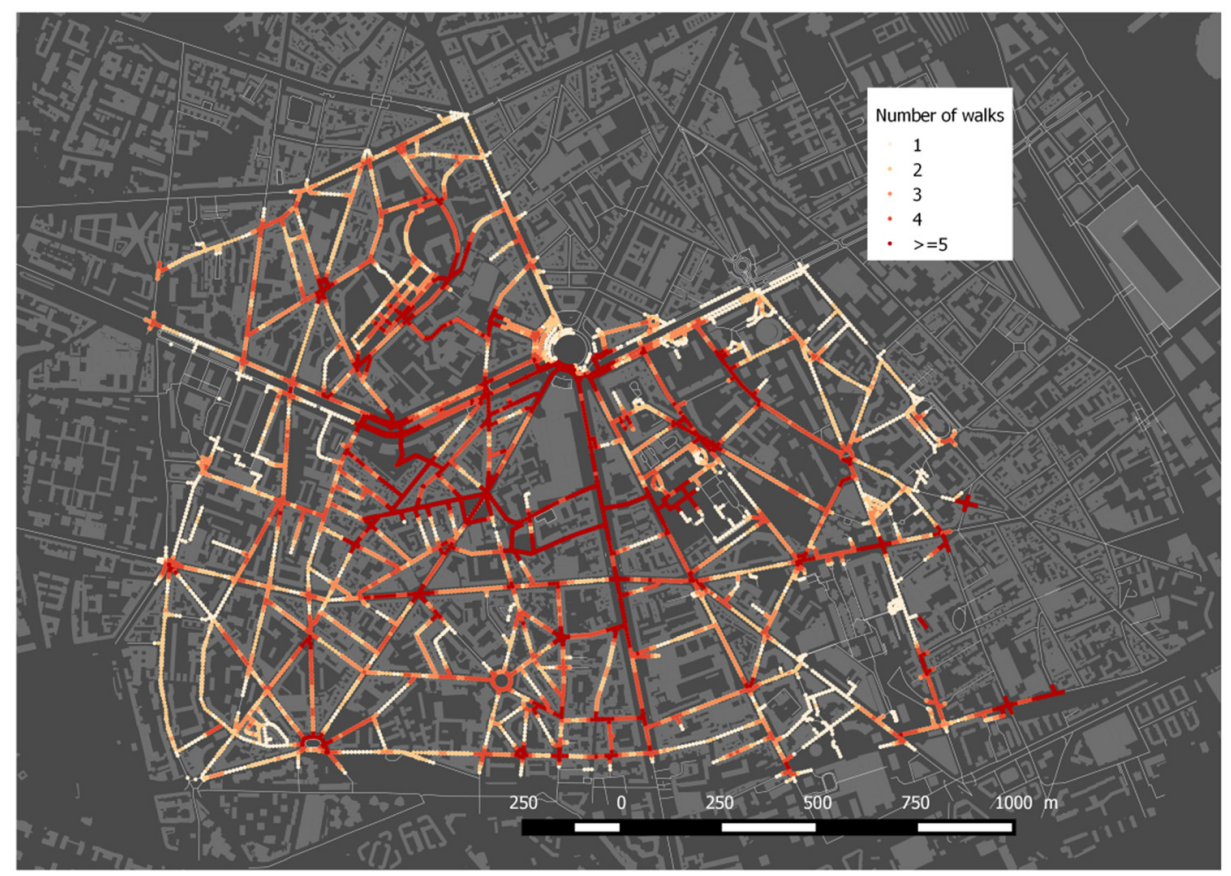

Figure 1 (color online) Number of passages at each location (only validated measurements).

\section{Map matching}

A GPS track is associated with each measurement session. However, the accuracy of the GPS data depends on many factors such as the quality of the GPS receiver, the characteristics of the surroundings such as the presence of high buildings, or the weather. In this study, the median standard deviation associated with the GPS locations was about 10 meters. Although rarely considered in studies dealing with geo-referenced mobile measurements, this can be problematic for the present analysis, because some measurements can be associated with an erroneous street.

Therefore, it was necessary to preprocess the GPS data, mapping each measurement to a location on the road, a problem commonly known as map-matching. For this study, a point-to-point method based was developed, on the basis of the following conditions: (i) all measurements were performed on sidewalks or on roads, and the sound levels measured on both sides of each street are considered to be equivalent and are snapped to the middle of the street; (ii) all GPS locations are snapped to the center of the closest street under the conditions that the operator walked with a maximum speed of $5 \mathrm{~km} / \mathrm{h}$ and that the map-matched 
point conserves the same direction of displacement (with a direction tolerance of $60^{\circ}$ ) as the original point; and (iii) the map-matched points are located at a maximum distance from the original GPS points equal to twice the standard deviation of the GPS tracker.

\section{E. $\quad$ From mobile measurements to observation locations}

In a previous study (31), it was shown that the median sound level $L_{50}$ is well correlated with the perceived loudness of an urban sound environment. The median sound level presents the advantage that it is less sensitive to peaks in the measurement than the energy-equivalent level. Exceptionally, peaks can be generated by the operators themselves, or can occur when extremely noisy vehicles pass by in the vicinity of the operators. In addition, the median sound level $L_{50}$ does not include A-weighting, which is known to reduce too much the influence of low frequencies $[63-500 \mathrm{~Hz}]$ at noise levels encountered in urban environments, and thus the influence of road traffic noise on overall perceived loudness. The first step in carrying out the spatial interpolation between measurement locations, was to aggregate, at each location, all mobile measurements that are within a radius $r$. This aggregation step is performed using the median sound level of all the 1-s values $L_{e q, 1 s}$ considered as independent observations. The associated value is assumed to be representative of the $L_{50}$ sound level at the observation location on weekdays and during the measurment periods [10-12h; 14-16h]. Every aggregated observation location is situated on the road network, because mobile measurements where only taken on sidewalks and pedestrian walkways in the public space. The road network that was used for map-matching was based on OpenStreetMap (32).

\section{F. $\quad$ Variogram and Kriging}

\section{Kriging method}

Number of statistical tools is provided by Geostatistics to analyze space variability and interpolate between measurements. Kriging algorithms give the best linear unbiased estimator and it is widely used for spatial interpolation of environmental domain such as hydrology, air pollution... In acoustics kriging method have been used lately

\section{Implementations and parameters}

The variogram and Kriging algorithms presented in this study are applied using the functions variog (computation of the variogram), variofit (best fit of the variogram) and krige.conv (Kriging function) of the packages GeoR (33) and GeoRcb (25). The empirical variogram is computed over a distance of 1000 meters. The classical estimator is chosen to compute the empirical variogram as defined in (34). The Matérn covariance model, as defined in (35), is used to compute the best fit of the experimental variogram with a fixed value for the shape parameter $\kappa=0.5$ and $\varphi$ the value of the range parameter to estimate.

\section{Euclidian vs cost-based distance}

The package GeoR has been used to interpolate using the Euclidian distance (EUCL). The package GeoRcb permits to use alternative definitions of distance. In this study, the distance between two observation locations has also been defined along the road network as presented in Figure 2. The error correlations in the traffic flow are assumed to be better modeled as a function of the distance along the road than the Euclidean distance. As a consequence, the use of the distance along the road network presumably allows to better model errors that come from the traffic. The distance between all the possible 
observation locations of the domain has been calculated with the Johnson's algorithm of the "distances" function of the package "igraph" of the R software described in (36).

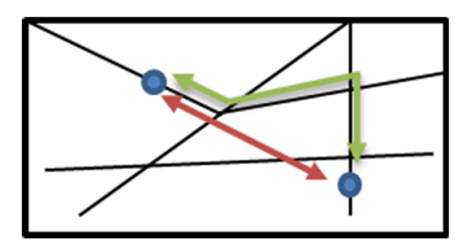

Figure 2(color online) Schema of the road network (shown in black), with the Euclidian Distance (shown in red) as well as the Road Network Distance (shown in green).

\section{Ordinary and universal Kriging}

Two Kriging methods are compared: Ordinary Kriging (OK) and Universal Kriging (UK). Universal Kriging is a variant of the ordinary Kriging operation that includes a linear trend. Barrigon et al. showed that urban noise is strongly stratified (18). Based on a similar statement, the linear trend $T$ in this study is defined as a linear regression based on 4 explanatory variables which are the distances $D_{i}$ between the observation locations of the study area and its closest roads belonging to 4 categories $i$. Equation 1 presents the equation of the trend:

$$
T \sim a . D_{1}+b . D_{2}+c . D_{3}+d . D_{4}+e
$$

in which $a, b, c, d$ and $e$ are adjusted constants.

Road categories have been defined based on the OpenStreetMap attributes as shown in Table 1. Figure 3 presents a map of the study area, in which the roads are shown in different colors according to their category. The first three categories correspond to streets with vehicular traffic, the last one to pedestrian streets. 


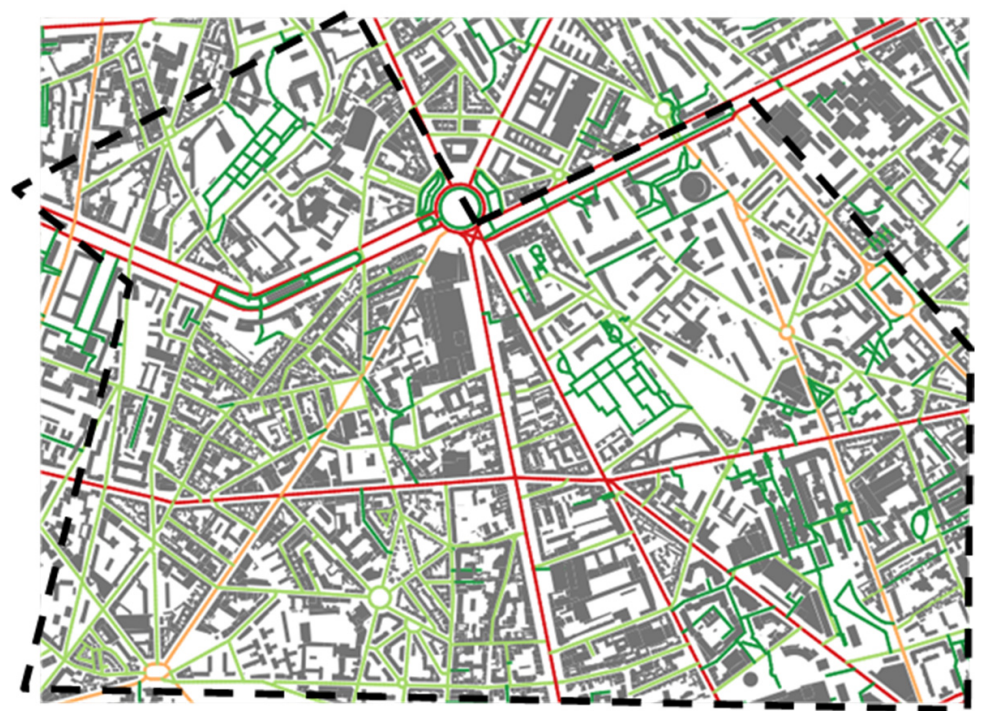

\begin{tabular}{|c|c|}
\hline $\begin{array}{l}\text { Open Street Map } \\
\text { attributes }\end{array}$ & Categories \\
\hline Primary & \multirow{5}{*}{1 (red) } \\
\hline Primary_link & \\
\hline Trunk & \\
\hline Secondary & \\
\hline Secondary link & \\
\hline Tertiary & \multirow{2}{*}{2 (orange) } \\
\hline Tertiary link & \\
\hline Living_street & \multirow{3}{*}{3 (light green) } \\
\hline Residential & \\
\hline Road & \\
\hline Service & \multirow{10}{*}{4 (dark green) } \\
\hline Service link & \\
\hline Steps & \\
\hline Crossing & \\
\hline Footway & \\
\hline Path & \\
\hline Pedestrian & \\
\hline Bridleway & \\
\hline Track & \\
\hline Unclassified & \\
\hline
\end{tabular}

Figure 3 (color online) Outline of the study area (black dashed line) and, in color, the 4 roads categories.

\section{G. Performance metrics}

206

207

208

209

210

The performance of the interpolation methods is assessed with two indicators: the Root Mean Square Error (RMSE) and the Pearson correlation coefficient (r) between the interpolated and the reference map. The geospatial interpolations are proposed following four methods: (a) ordinary Kriging (OK + EUCL), (b) ordinary Kriging using the distance along the road network (OK + ROAD), (c) universal Kriging (UK + EUCL), (d) universal Kriging using the distance along the road network (UK + ROAD).

\section{III.Results}

212

\section{A. $\quad$ Reference sound map}

A reference noise map based on all mobile measurements is first computed. For each observation location, the mobile measurements that are within a specific integration radius are mapped to this location. A sensitivity analysis of the spatial representativeness and the expected accuracy of the sound level at the aggregation radius and the aggregated number of 1-s samples is then performed.

Six values for the aggregation radius have been tested: $2.5 \mathrm{~m}, 5 \mathrm{~m}, 10 \mathrm{~m}, 15 \mathrm{~m}, 25 \mathrm{~m}$ and $50 \mathrm{~m}$. For each radius value, a set of observation locations has been selected, uniformly distributed. For this statistical analysis, the observation locations are spaced apart by at least two times the radius value, to avoid redundancy. In Figure 4, three of those subsets of observation locations are shown.
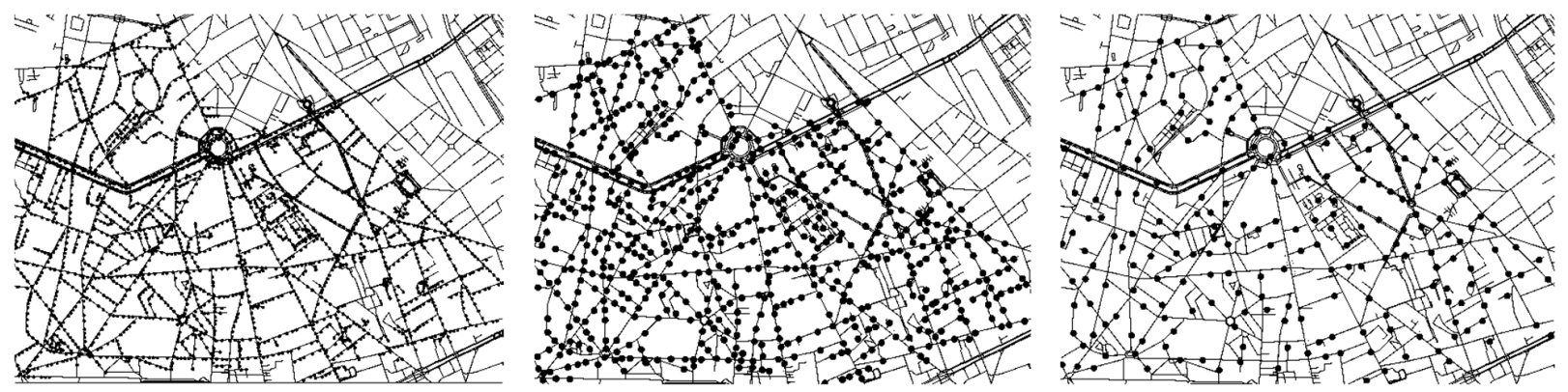
(a)

(b)

(c)

Figure 4 Example of three selected sets of observation locations (dots) for radii: (a) $2.5 \mathrm{~m}$, (b) $15 \mathrm{~m}$ and (c) $50 \mathrm{~m}$. Observation locations are located on the study area road network (solid lines).

Figure 5(a) shows the distribution of the aggregated sound levels $L_{50}$ over the study area for the smallest and largest aggregation radii $(2.5 \mathrm{~m}$ and $50 \mathrm{~m})$ as presented in Section II.E. Figure 5 (b) shows the $P_{90}-P_{10}$ indicator (where $P_{10}$ and $P_{90}$ are the percentiles 10 and 90 of the sound level distributions), which reflects the width of these distributions for all the studied aggregation radii. As expected, the range of the aggregated sound levels $L_{50}$ over the study area decreases as the value of the radius increases. More prosaically, the reference sound map will appear to be blurred. A small radius will give rise to a more detailed reference sound map, but comes with a decreased number of measurements at each location that may therefore no longer be representative. The results show that the influence of the aggregation radius over the sound level distribution is relatively small, with $P_{90}-P_{10}$ decreasing from $13.5 \mathrm{~dB}$ to $10.5 \mathrm{~dB}$.

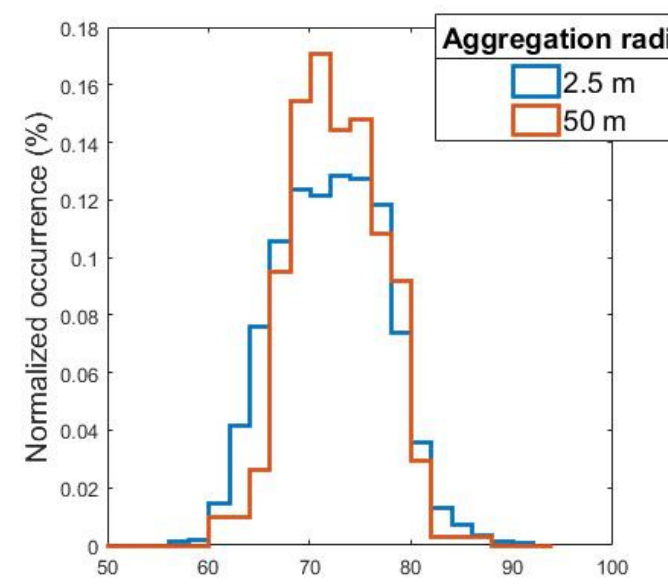

(a) Sound levels distribution $(\mathrm{dB})$

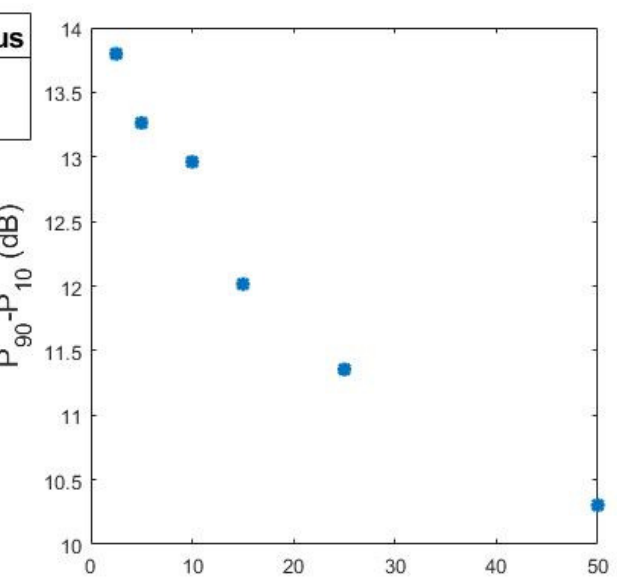

(b) Aggregation radius ( $m$ )

Figure 5 (color online) (a) Distribution of the aggregated sound levels $L_{50}$ over the study area for two values of the aggregation radius. (b) Width of the distribution of the sound levels $\left(P_{90}-P_{10}\right)$ for 6 different aggregation radii

A bootstrapping method (bootstrp function, statistical toolbox, Matlab) is proposed to analyze the sensitivity of the $L_{50}$ value to the aggregation radius and the number of measurements. This method relies on random sampling, with replacement, of the 1-s measurements for each location within the study area (37). Multiple replications of the method permits to compute the variance associated with the average $L_{50}$ value at each observation location due to the sample characteristics. Figure 6 presents (a) the standard deviation of 1000 bootstrap replications of the calculated $L_{50}$ varying with the number of 1-s samples and (b) the relationship between the aggregation radius and the proportion of retained locations, considering a minimum number of 1 -second samples of 180 . The correlation between these two parameters is statistically significant $(\mathrm{r}=0.53, \mathrm{p}<.05)$, but a large aggregation radius does not always imply a large number of measurements (e.g., along the borders of the study area) and vice versa (e.g., if the operator measured a few minutes at one specific location). 


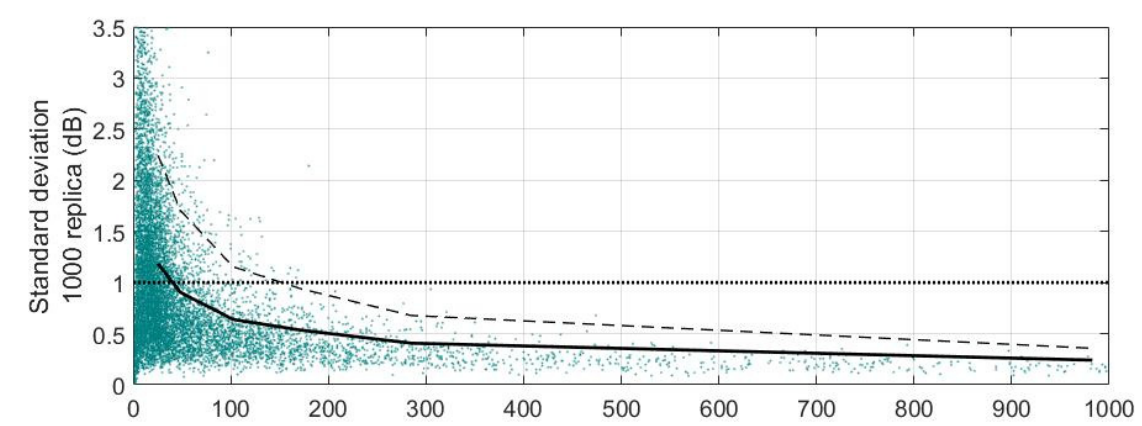

(a) Number of 1-s sample (sec)

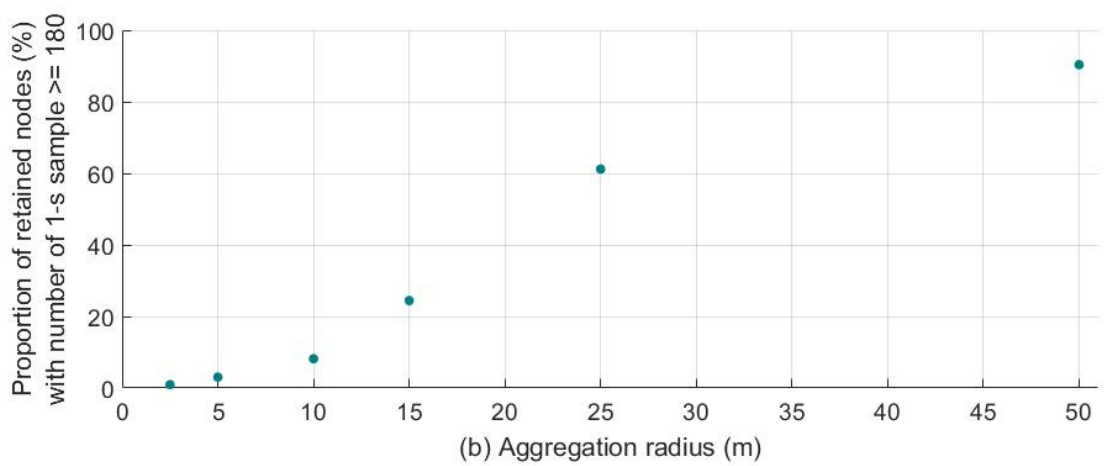

Figure 6 (color online) The upper plot shows the relationship between the standard deviation of 1000 replications (subsets) of the underlying reference data set and the number of 1-s samples of the subsets. The lower plot shows the relationship between the aggregation radius and the proportion of retained measurement locations, considering a minimum number of 1 -second samples of 180. Each dot represents a replication, the solid line denotes the mean value, the dashed line denotes the percentile-90).

The variation specific to each locations is found to be considerably smaller than the global variation between the locations over the whole study area, which is about $5 \mathrm{~dB}$ (see Figure 5). If we consider a standard deviation smaller than $1 \mathrm{~dB}$ as acceptable, $90 \%$ of the calculated standard deviations are below this threshold when a sample is composed by a minimum of 1801 -s measurements. The chosen threshold also guarantees to have enough data to carry out the spatial analysis. An aggregation radius of 25 meters is chosen as the threshold value because it permits to retain more than $60 \%$ of the computed locations. This aggregation radius also has the advantage that it corresponds to the longitudinal spatial representativeness of sound level measurements found in literature, perceptually (38) or physically (24), thus suggesting that the resulting sound level map will not be too sparse.

On the basis of the above results, it can be concluded that, at least for the urban study area considered, three-minute measurements provide sufficient confidence in the aggregated measurement value, even if its representativeness is not guaranteed. As the purpose of the present study was to perform a comparison of interpolation techniques, the calculated noise maps only need to respect the spatial variation of the sound level, but do not necessarily need to be representative of a homogeneous time period.

Figure 7 presents the resulting reference map of the median sound level $\left(L_{50}, \mathrm{~dB}\right)$. For the calculation of this map, a distance of $10 \mathrm{~m}$ between each observation location was used, resulting in 4360 locations. 


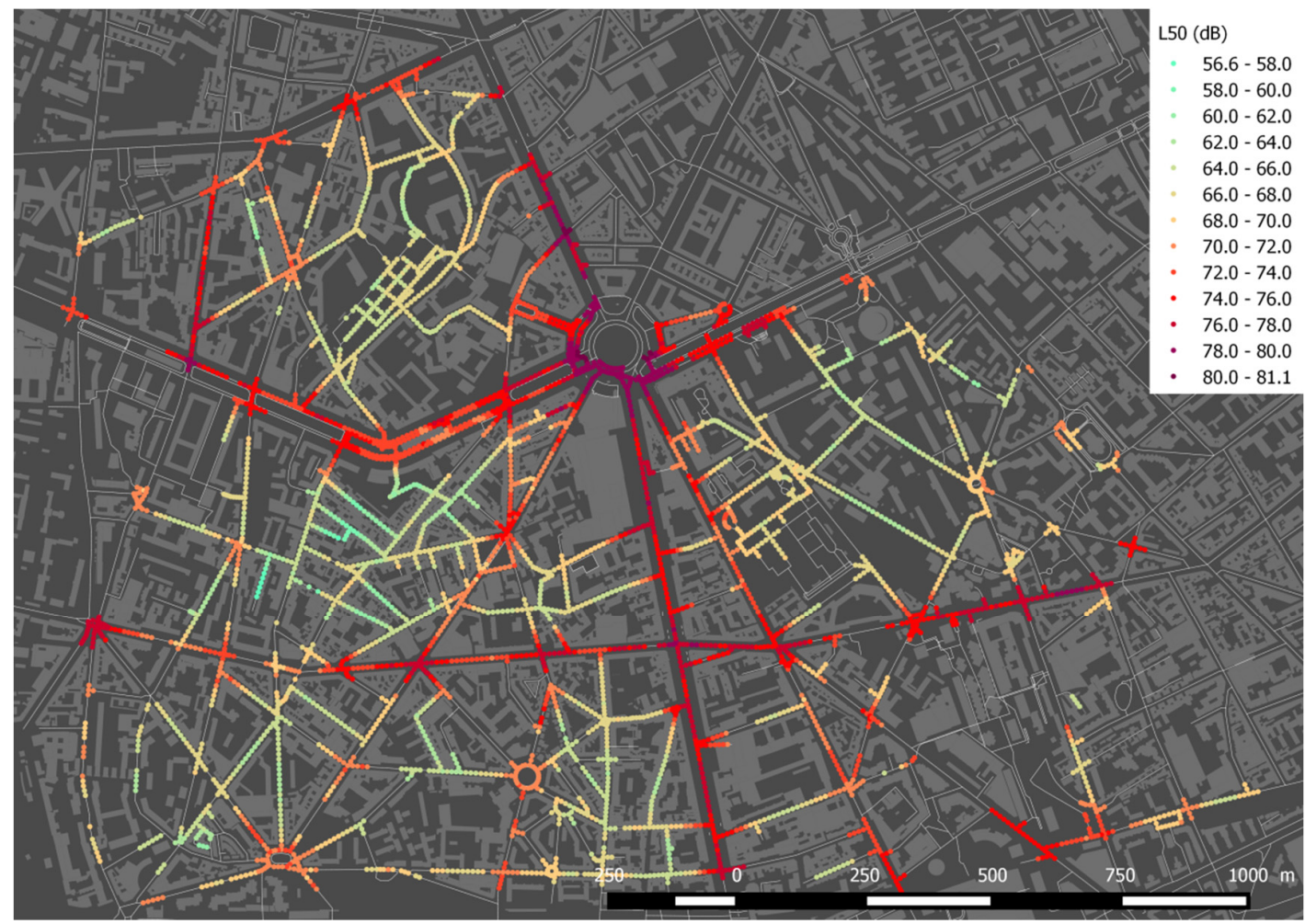

Figure 7 (color online) Reference sound map, integration radius 25 meters, integration time $>180 \mathrm{sec}$. $(\mathrm{n}=4360)$

\section{$271 \quad$ B. Spatial dependence of the data}

272 The spatial dependence of the data is highlighted through the calculation of variograms, which express the semivariance between $L_{50}$ values for a couple of locations according to their distance. On Figure 8, four fitted variograms derived from the reference sound map are presented: (a) an ordinary variogram with the Euclidean distance $(\mathrm{OK}+\mathrm{EUCL})$, (b) an ordinary variogram using the distance along the road network $(\mathrm{OK}+\mathrm{ROAD})(\mathrm{c})$ an universal variogram which accounts for the trend described in Section II with the Euclidean distance (UK+EUCL), (d) an universal variogram which accounts for the trend and also uses

278 the distance along the road network (UK+ROAD). 


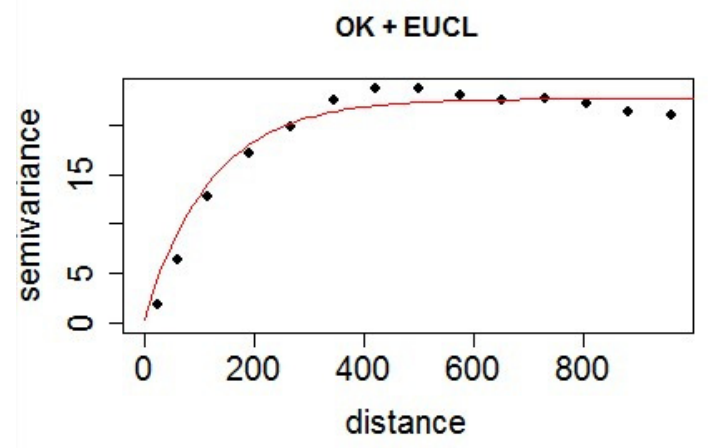

OK + ROAD

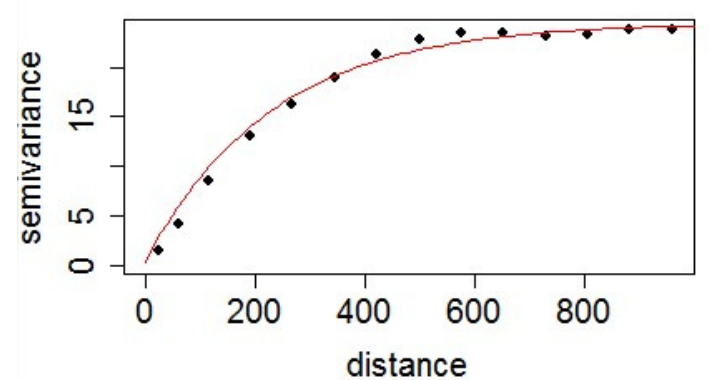

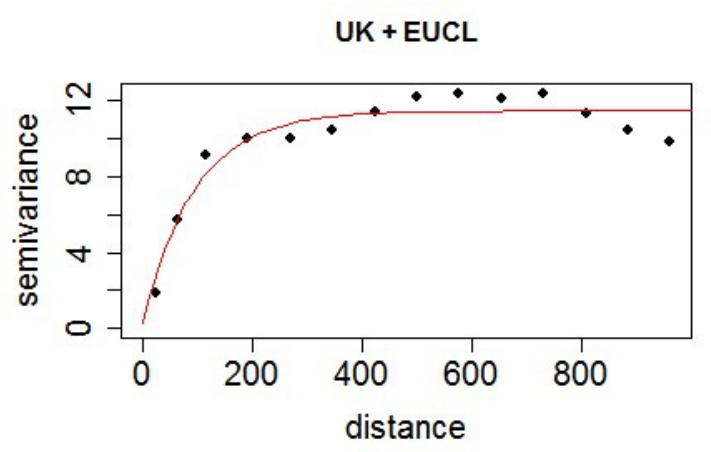

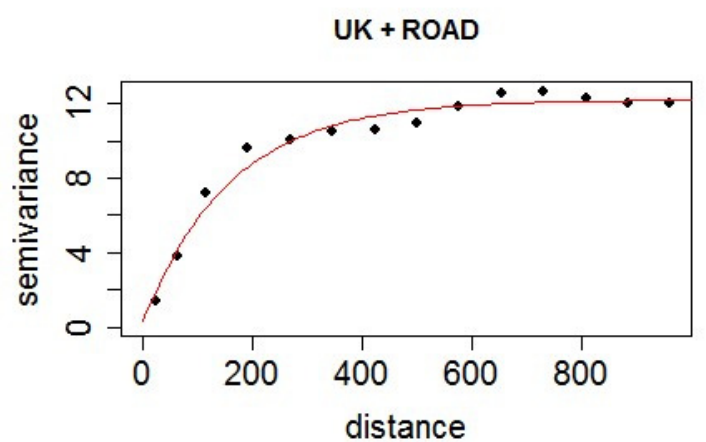

\begin{abstract}
Figure 8 Empirical variograms (dots) and best fitted parametrical models (red line) computed using the ordinary Kriging (OK) and universal Kriging (UK) methods. The printed distance is computed using the Euclidian distance (EUCL) or the distance along the road network (ROAD) (distance in meters, and semivariance in $\mathrm{dB}^{2}$ ).
\end{abstract}

The parameters of the best fitted covariance models are presented in Table 1. The practical ranges of the variograms, defined as the value for which the correlation function decays to $5 \%$ of its value at 0 , are (a) $366 \mathrm{~m}$ for OK+EUCL (b) $285 \mathrm{~m}$ for UK+EUCL (c) $691 \mathrm{~m}$ for OK+ ROAD (d) $481 \mathrm{~m}$ for UK+ROAD. For OK+EUCL, hardly any information is given by an observation to an estimated value located in a radius superior to $366 \mathrm{~m}$. As the nugget variance $\tau^{2}$ is null, the variogram asymptote, or sill value, corresponds to the signal variance $\sigma^{2}$. This value at 1000 meters is $22.4 \mathrm{~dB}^{2}$ and $24.2 \mathrm{~dB}^{2}$ for OK + EUCL and $\mathrm{OK}+\mathrm{ROAD}$ methods, and $11.2 \mathrm{~dB}^{2}$ and $11.9 \mathrm{~dB}^{2}$ for UK + EUCL and UK + ROAD. Thus, adding the trend, defined in Section II, permits to drastically reduce the signal variance and illustrates the strong correlation between the urban sound levels and the proximity to different types of roads. Also, the practical range of the variograms increases when the alternative definition of distance from the road network is used (from 366 to $691 \mathrm{~m}$ and from 285 to $481 \mathrm{~m}$ ).

Table 1 - Parameters of the Kriging methods

\begin{tabular}{cccccc}
\hline Kriging method & Covariance model & $\begin{array}{c}\text { Nugget } \\
\text { variance } \\
\left(\mathrm{dB}^{2}\right)\end{array}$ & $\begin{array}{c}\text { Sill } \\
\left(\mathrm{dB}^{2}\right)\end{array}$ & $\begin{array}{c}\text { Range } \\
\text { parameter } \\
(\mathrm{m})\end{array}$ & $\begin{array}{c}\text { Practical Range } \\
(\mathrm{m})\end{array}$ \\
\hline OK+EUCL & Matérn with fixed $\kappa=0.5$ & 0 & 22.4 & 122.2 & 366 \\
UK+EUCL & Matérn with fixed $\kappa=0.5$ & 0 & 11.2 & 95.1 & 285 \\
OK+ROAD & Matérn with fixed $\kappa=0.5$ & 0 & 24.2 & 230.7 & 691 \\
UK+ROAD & Matérn with fixed $\kappa=0.5$ & 0 & 11.9 & 160.6 & 481
\end{tabular}


A subset of the observation locations is selected from the reference map and is interpolated over the whole study area using the four tested strategies. As described in Section IV, the median sound level values at the observation locations are computed from at least $180 L_{\text {eq, ls }}$ measurements included in a $25 \mathrm{~m}$ radius around each observation location. The subset of observation locations for evaluation is randomly selected and 1000 replications are performed for each subset configuration. The replications give information about the variability due to a chosen set of observation nodes. Figure 9 presents the relationships between two indicators of performance (RMSE and the Pearson correlation coefficient), and the density of nodes (number of nodes per sq. $\mathrm{km}$ ) for the four methods of interpolation.

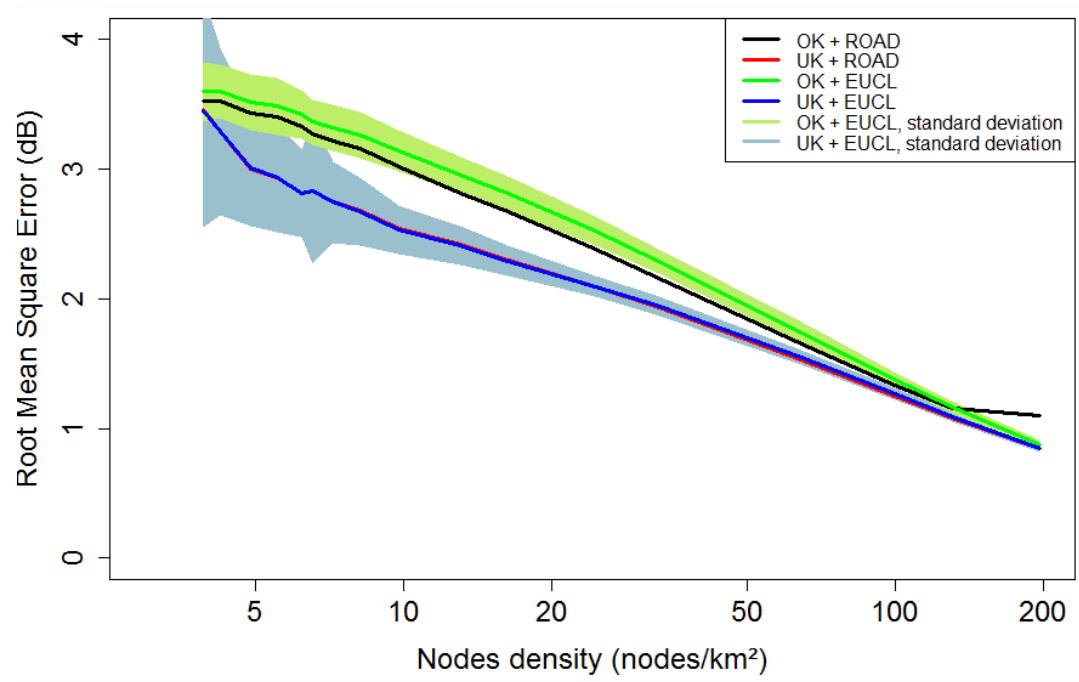

(a)

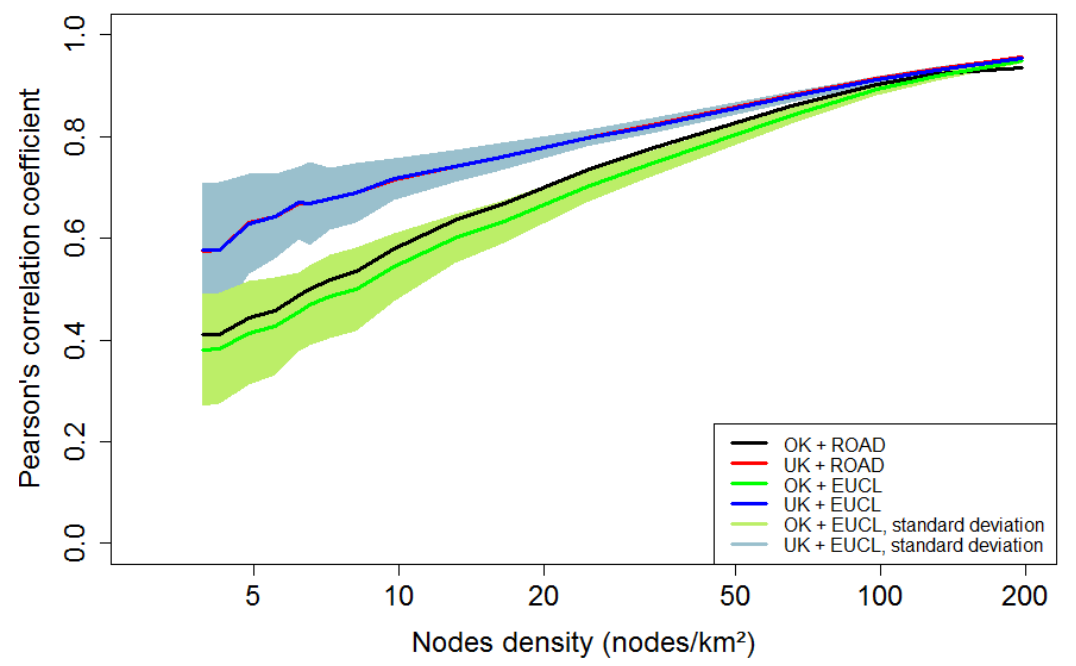

(b) nodes (number of nodes per sq. km) for the four methods of interpolation. The area corresponds to the standard deviation associated with the 1000 
Universal Kriging (UK) drastically increases the quality of the results on both indicators compared to ordinary Kriging (OK). Taking into account the distance along the road network (ROAD) only leads to better results for the ordinary Kriging cases.

From a practical point of view, in case only fixed measurement stations are used, 15 observation locations per sq. km is already a large number. In this case, Figure 9 shows that the correlation between interpolated and reference sound levels is between 0.5 and 0.8 , and that the RMSE value is between 2.5 and $3.5 \mathrm{~dB}$. Figure 10 shows an interpolated sound map, using the UK + EUCL and the OK + EUCL methods, based on one of the random sets of 42 observation locations (15 locations per sq. $\mathrm{km}$ ). The associated prediction standard error maps are also given, as the Kriging methods give access to this information (35). Figure 10 shows a good correspondence with the reference sound map (see Figure 7). Nevertheless, the dispersion in Figure 9 shows that the observation locations have an important influence 321 over the performance of the algorithm.

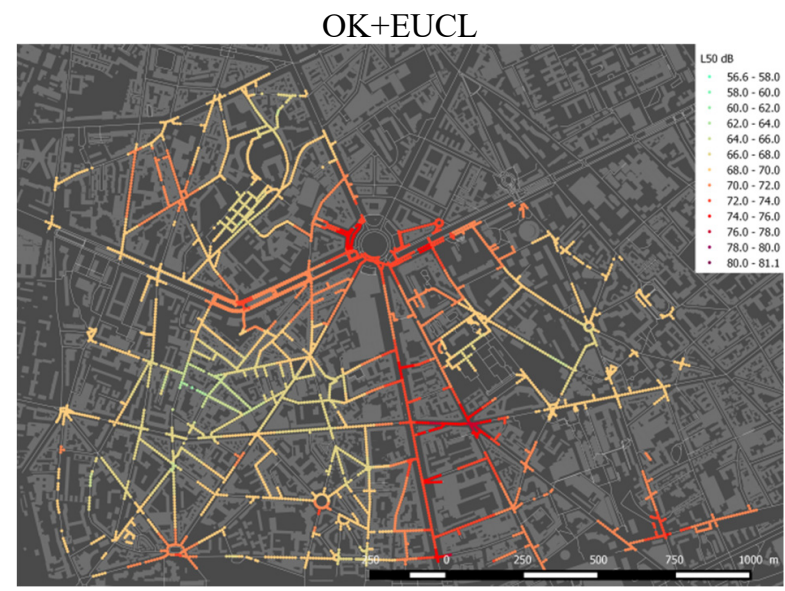

(a)

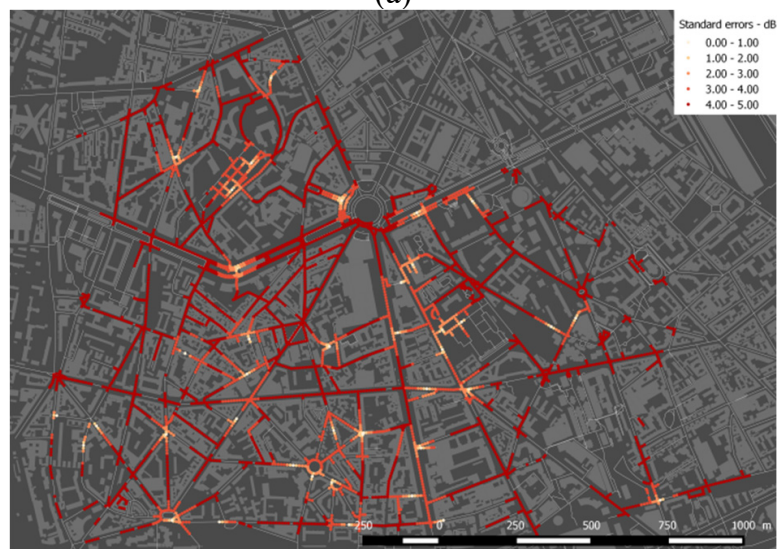

(c)

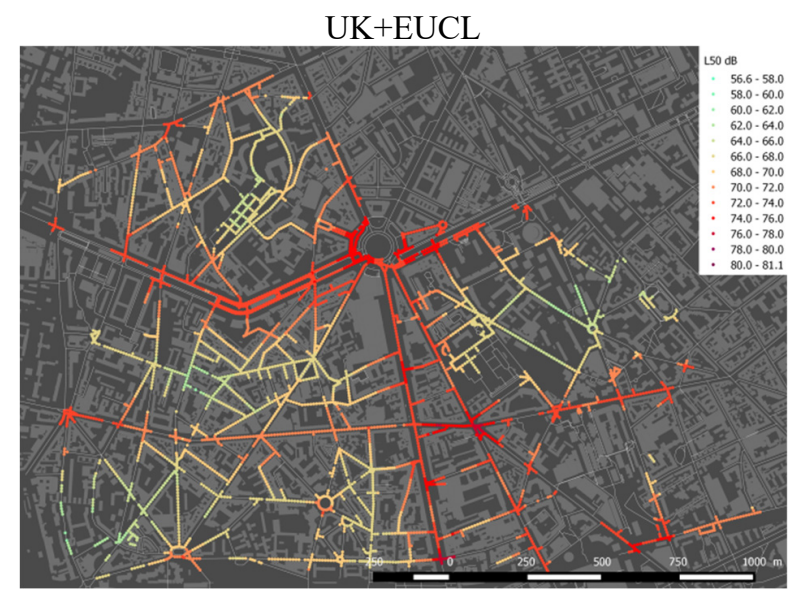

(b)

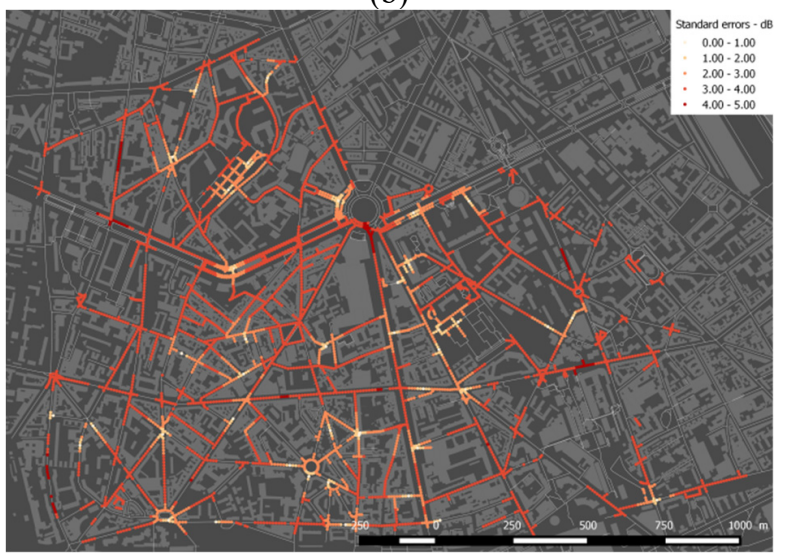

(d)

Figure 10 (color online) Example of an interpolated sound map from 42 observations: Estimated $\mathrm{L}_{50}$ values using the (a) OK+EUCL and (b) UK+EUCL Kriging methods, and the associated standard errors maps (c) OK+EUCL and (d) UK+EUCL

Figure 11 shows the average errors for the 1000 replications (42 observation locations), against the reference map presented in Figure 7. Figure 11 (a-d) shows that all the interpolation strategies 


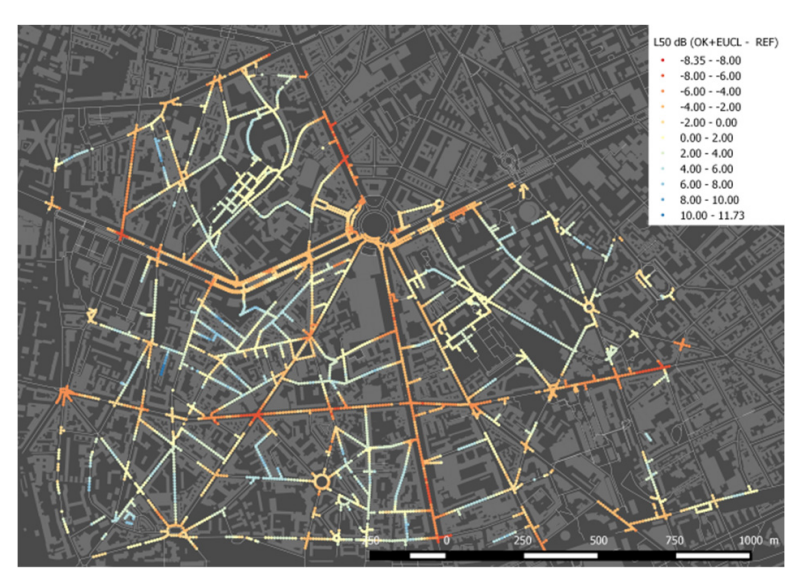

(a) $(\mathrm{OK}+\mathrm{EUCL})$ - REF

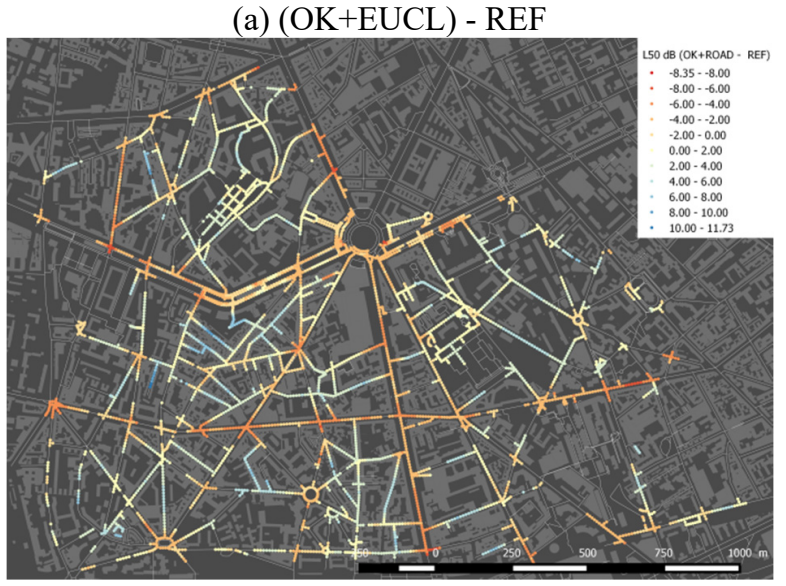

(c) $(\mathrm{OK}+\mathrm{ROAD})$ - REF

underestimate the sound level for large boulevards and overestimate the sound level for quiet places, but this is a common limitation of interpolation methods. This is less pronounced for universal Kriging (Figure 11 (b)). In this case, the trend partly corrects this shortcoming, and the errors are distributed more uniformly over the study area. A comparison between Figure 11(a) and (c) shows the difference between the use of the Euclidian and the road network distance; no major influence is observable.

Figure 11 (color online) Average errors for 1000 replications (42 observation locations) against the reference map REF for the four interpolation

methods (a) OK+EUCL, (b) UK+EUCL, (c) OK+ROAD and (d) UK+ROAD.

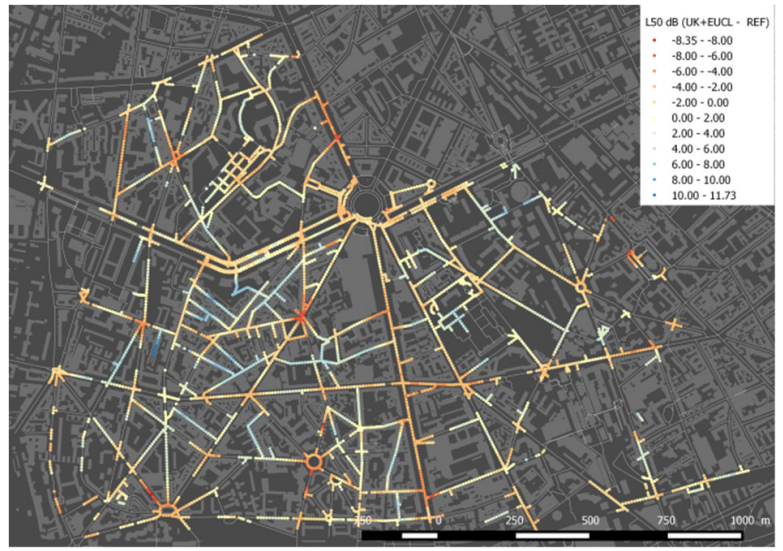

(b) $(\mathrm{UK}+\mathrm{EUCL})-\mathrm{REF}$

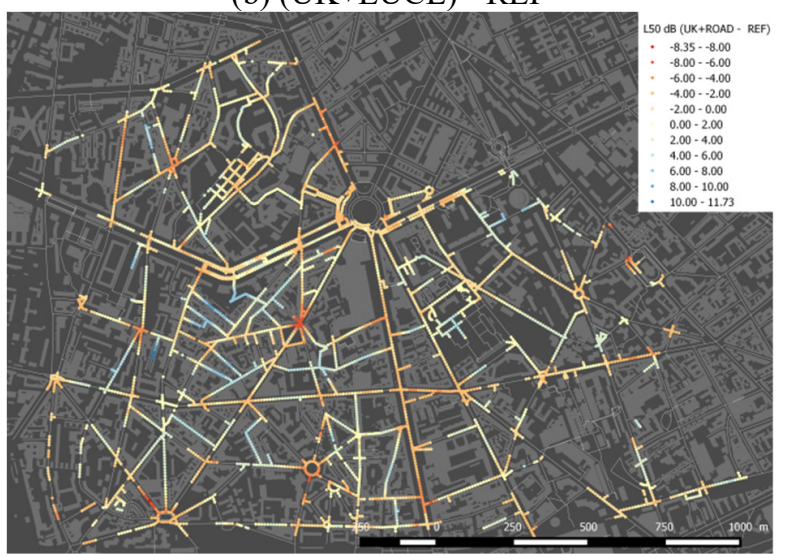

(d) $(\mathrm{UK}+\mathrm{ROAD})-\mathrm{REF}$

Figure 9 shows that the standard deviation of the performance indicators can be important, especially in case of a small density of measurement locations. In order to identify the principal factors which influence the performance of the Kriging algorithms, a short statistical analysis has been performed over geospatial indicators of the spatial distribution of 42 observations. Table 2 presents some of the parameters which have been calculated for each generated set of observations.

The Variance Mean Ratio (VMR), also called index of dispersion, is an indicator of a good dispersion of the observations over the domain. For this, the domain is divided in $6 \times 6$ cells. In each cell, the number of observations is calculated. The variance mean ratio corresponds to the ratio between (i) the variance of the number of observations between each cell, and (ii) the mean number of observations for a cell. If $\mathrm{VMR}$ is equal to 1 , the observations are randomly dispersed; if $\mathrm{VMR}<1$ the observations are more 
dispersed than random (e.g., regular distribution); if VMR $>1$ the observations are more clustered than a random dispersion.

MEAN_ROAD, STD_ROAD, KURT_ROAD, SKEW_ROAD are indicators of the distribution of the observations over the road categories (integers from 1 to 4, see Figure 2). For example, if MEAN_ROAD is equal to 1 and STD_ROAD equal to 0 , it means that all the observations are located over roads of type 1 (avenue/boulevard).

Finally, the CENTER indicator represents the distance between the center of gravity of the observation locations and the center of gravity of the whole road network within the case study area. If CENTER is small, the observations are well centered over the domain.

Table 2 - Geospatial indicators of the observation locations.

\begin{tabular}{lll}
\hline Index & Name & Range \\
\hline VMR & Variance Mean Ratio & {$[0.8-1.9]$} \\
STD_L50 & Standard deviation on observed sound levels $\left(\mathrm{L}_{50}\right)$ & {$[2.8-6](\mathrm{dB})$} \\
& & {$[1.7-2.8]$} \\
MEAN_ROAD & Average closest road categories & {$[0.5-1.2]$} \\
STD_ROAD & Standard deviation on closest road categories & {$[-0.19-1.6]$} \\
KURT_ROAD & Kurtosis closest road categories & {$[1.4-7.4]$} \\
SKEW_ROAD & Skewness closest road categories & {$[0.34-364](\mathrm{m})$} \\
CENTER & $\begin{array}{l}\text { Distance between the gravity center of the observations and } \\
\text { the gravity center of the whole network }\end{array}$ &
\end{tabular}

Table 3 presents the Pearson correlation coefficient between the spatial distribution indicators and the performance indicators. It shows that the STD_L50 is the only geospatial indicator that is well correlated with the performance indicators, particularly for ordinary Kriging. As it can be expected, this suggests that the measurement stations should be placed in locations that represent a large distribution of sound levels. For universal Kriging, this factor is less important because of the correction brought by the trends. The same analysis was carried out with other performance metrics than the Pearson correlation coefficient, but this did not bring more information.

Table 3 - Pearson correlation coefficient between the geospatial and performance indicators for two Kriging methods $(* \mathrm{p}<0.05)$.

\begin{tabular}{lcccc}
\hline & $\mathrm{r}$ & $\mathrm{r}$ & $\mathrm{RMSE}$ & $\mathrm{RMSE}$ \\
& $(\mathrm{OK}+\mathrm{EUCL})$ & $(\mathrm{UK}+\mathrm{EUCL})$ & $(\mathrm{OK}+\mathrm{EUCL})$ & $(\mathrm{UK}+\mathrm{EUCL})$ \\
\hline VMR & $0.06^{*}$ & & $-0.07^{*}$ & \\
STD_L50 & $\mathbf{0 . 4 8}^{*}$ & $\mathbf{0 . 2 7 *}$ & $\mathbf{- 0 . 5 5}^{*}$ & $\mathbf{- 0 . 2 1 ^ { * }}$ \\
MEAN_ROAD & $0.07^{*}$ & & & $-0.04^{*}$ \\
STD_ROAD & $0.19^{*}$ & $0.07^{*}$ & $-0.19^{*}$ & $-0.10^{*}$ \\
KURT_ROAD & $-0.10^{*}$ & $-0.06^{*}$ & $0.07^{*}$ & $0.09^{*}$ \\
SKEW_ROAD & $-0.16^{*}$ & $-0.07^{*}$ & $0.14^{*}$ & $0.11^{*}$ \\
CENTER & $-0.05^{*}$ & & $0.06^{*}$ &
\end{tabular}

\section{IV.Discussion}

As the study area was the XIII ${ }^{\text {th }}$ district of Paris, the variogram and Kriging parameters were only adjusted for this district. Even if some of these parameters are comparable with those from previous studies (24), other replications in other urban contexts will be necessary to extend the conclusions of this work. 
On the one hand, the alternative definition of distance along the road network slightly increases the performance of the algorithms, but only for ordinary Kriging methods. On the other hand, the proposed trend based on the distance to the closest roads by category, as defined in Section II, strongly improves the results for all Kriging methods. These results confirm the observations done in $(18,39,40)$ which state that using a street categorization method that accounts for street use is particularly appropriate to study the spatial variability of urban sound levels. Thus, to continue to improve the performance of the interpolation methods, efforts should be focused on the trend definition. Nevertheless, a complex trend definition is comparable to the computation of a model-based noise map. Moreover, it might increase the computational cost of the interpolation method considerable, and can possibly introduce new error sources. It can also imply to previously generate the sound map of the city, modeling all relevant sound sources which some are not usually included and naturally imply the fusion of the same indicators which also have to be computed.

The errors associated with the observations are not taken into account in our Kriging approach; nevertheless, a follow-up study should investigate the integration of noisy observations with a proper uncertainty associated with each measurement location. Maybe data assimilation methods used in geosciences, e.g., computing the so-called best linear unbiased method $(41,42)$, could be useful to achieve this task.

All the presented results are valid for one homogeneous time period. Time interpolation could be added. This interpolation could be done in pre- or post-processing, relying on previous works dealing with temporal interpolation of urban noise levels (43) or directly with a spatio-temporal Kriging.

Finally, this study shows the influence of the spread of observation locations over the study area to correctly interpolate sound levels. As it can be expected, the results suggest that fixed measurement stations should be placed to obtain a large distribution of sound levels and so, a large variety of sound environments. Nevertheless, even if it can be estimated, this information is not fully available prior to the installation of the measurement stations. Other indicators, such as the distribution of the measurement stations over the various road categories, or the location of the center of gravity of the measurement stations, which are available a priori, do not show relevant relationship with performance indicators.

The main conclusions are:

- A minimum of 180 1-s measurements are needed to obtain an acceptable level of confidence $(1 \mathrm{~dB})$ in the $\mathrm{L}_{50}$ value calculated at each location within the study area, with an aggregation radius of $25 \mathrm{~m}$. 
- The practical ranges of the variograms computed for the four Kriging methods are between 250 and 700 meters.

- Using the distance along the road network in the Kriging method drastically increases the performance in case of ordinary Kriging, but not in case of universal Kriging.

- Universal Kriging, which consists of adding a local trend in the ordinary Kriging formulation, is a promising method. Nevertheless, it introduces an additional calculation of the trend that has a computational cost and can in itself be a source of error.

- Approximately 50 observation locations per sq. $\mathrm{km}$ are needed in order to get a correlation coefficient superior to 0.8 and a RMSE value inferior to $2.5 \mathrm{~dB}$ between the reference and the interpolated map.

In view of the large density of observation locations needed to obtain a noise map with a high accuracy and the strong improvement brought by the trend in the Kriging formulation, further studies should probably focus on fusion or assimilation techniques combining measurements and numerical simulation results.

\section{Acknowledgments}

This work was carried out in the framework of the GRAFIC project, supported by the French Environment and Energy Management Agency (ADEME) under contract No. 1317C0028. The authors also would like to thank the BruitParif team which completed most of the measurements.

\section{References}

1. EC. Directive 2002/49/EC of the European parliament and the Council of 25 June 2002 relating to the assessment and management of environmental noise. Off J Eur Communities. 2002;189(12):12-25.

2. Kephalopoulos S, Paviotti M, Anfosso-Lédée F, Van Maercke D, Shilton S, Jones N. Advances in the development of common noise assessment methods in Europe: The CNOSSOS-EU framework for strategic environmental noise mapping. Sci Total Environ. 2014 Jun;482-483:400-10.

3. Zambon G, Benocci R, Bisceglie A, Roman HE, Bellucci P. The LIFE DYNAMAP project: Towards a procedure for dynamic noise mapping in urban areas. Appl Acoust. 2017 Sep;124:52-60.

4. Asensio C. Acoustics in Smart Cities. Appl Acoust. 2017 Feb;117, Part B:191-2.

5. Hong JY, Jeon JY. Exploring spatial relationships among soundscape variables in urban areas: A spatial statistical modelling approach. Landsc Urban Plan. 2017 Jan;157:352-64.

6. Harman $\mathrm{BI}$, Koseoglu $\mathrm{H}$, Yigit $\mathrm{CO}$. Performance evaluation of IDW, Kriging and multiquadric interpolation methods in producing noise mapping: A case study at the city of Isparta, Turkey. Appl Acoust. 2016 Nov;112:147-57.

7. Mydlarz C, Salamon J, Bello JP. The implementation of low-cost urban acoustic monitoring devices. Appl Acoust. 2017 Feb;117, Part B:207-18. 
8. Sevillano X, Socoró JC, Alías F, Bellucci P, Peruzzi L, Radaelli S, et al. DYNAMAP - Development of low cost sensors networks for real time noise mapping. Noise Mapp. 2016;3(1).

9. Aspuru I, García I, Herranz K, Santander A. CITI-SENSE: methods and tools for empowering citizens to observe acoustic comfort in outdoor public spaces. Noise Mapp. 2016 Jan;3(1):37-48.

10. Guillaume G, Can A, Petit G, Fortin N, Palominos S, Gauvreau B, et al. Noise mapping based on participative measurements. Noise Mapp. 2016 Jan;3(1):140-56.

11. Maisonneuve N, Stevens M, Niessen ME, Steels L. NoiseTube: Measuring and mapping noise pollution with mobile phones. Environ Sci Eng Subser Environ Sci. 2009;215-28.

12. Issarny V, Mallet V, Nguyen K, Raverdy P-G, Rebhi F, Ventura R. Dos and Don'ts in Mobile Phone Sensing Middleware: Learning from a Large-Scale Experiment. In 2016.

13. Geraghty D, O'Mahony M. Investigating the temporal variability of noise in an urban environment. Int J Sustain Built Environ. 2016 Jun;5(1):34-45.

14. Barrigón Morillas JM, Prieto Gajardo C. Uncertainty evaluation of continuous noise sampling. Appl Acoust. 2014 Jan;75:27-36.

15. Zuo F, Li Y, Johnson S, Johnson J, Varughese S, Copes R, et al. Temporal and spatial variability of traffic-related noise in the City of Toronto, Canada. Sci Total Environ. 2014 Feb 15;472(Supplement C):1100-7.

16. Brocolini L, Lavandier C, Quoy M, Ribeiro C. Measurements of acoustic environments for urban soundscapes: choice of homogeneous periods, optimization of durations, and selection of indicators. J Acoust Soc Am. 2013;134(1):813-21.

17. Prieto Gajardo C, Barrigón Morillas JM. Stabilisation patterns of hourly urban sound levels. Environ Monit Assess. 2015 Jan;187(1):4072.

18. Morillas JMB, Escobar VG, Sierra JAM, Vílchez-Gómez R, Vaquero JM, Carmona JT. A categorization method applied to the study of urban road traffic noise. J Acoust Soc Am. 2005 May;117(5):284452.

19. Can A, Van Renterghem T, Rademaker M, Dauwe S, Thomas P, De Baets B, et al. Sampling approaches to predict urban street noise levels using fixed and temporary microphones. J Environ Monit JEM. 2011 Oct;13(10):2710-9.

20. Mateus M, Dias Carrilho JA, Gameiro da Silva MC. Assessing the influence of the sampling strategy on the uncertainty of environmental noise measurements through the bootstrap method. Appl Acoust. 2015 Mar 1;89(Supplement C):159-65.

21. Liu J, Kang J, Luo T, Behm H, Coppack T. Spatiotemporal variability of soundscapes in a multiple functional urban area. Landsc Urban Plan. 2013;115:1-9. 
22. Segura Garcia J, Pérez Solano JJ, Cobos Serrano M, Navarro Camba EA, Felici Castell S, Soriano Asensi A, et al. Spatial Statistical Analysis of Urban Noise Data from a WASN Gathered by an IoT System: Application to a Small City. Appl Sci. 2016 Nov 26;6(12):380.

23. Huang B, Pan Z, Yang H, Hou G, Wei W. Optimizing stations location for urban noise continuous intelligent monitoring. Appl Acoust. 2017 Dec 1;127:250-9.

24. Can A, Dekoninck L, Botteldooren D. Measurement network for urban noise assessment: Comparison of mobile measurements and spatial interpolation approaches. Appl Acoust. 2014 Sep;83:32-9.

25. López-Quílez A, Muñoz F. Geostatistical computing of acoustic maps in the presence of barriers. Math Comput Model. 2009 Sep 1;50(5):929-38.

26. Hachem S, Mallet V, Ventura R, Pathak A, Issarny V, Raverdy PG, et al. Monitoring Noise Pollution Using the Urban Civics Middleware. In: 2015 IEEE First International Conference on Big Data Computing Service and Applications. 2015. p. 52-61.

27. Wei W, Van Renterghem T, De Coensel B, Botteldooren D. Dynamic noise mapping: A map-based interpolation between noise measurements with high temporal resolution. Appl Acoust. 2016;101:127-40.

28. Ventura R, Mallet V, Issarny V, Raverdy P-G, Rebhi F. Estimation of urban noise with the assimilation of observations crowdsensed by the mobile application Ambiciti. In Hong-Kong; 2017.

29. Aletta F, Kang J. Soundscape approach integrating noise mapping techniques: a case study in Brighton, UK. Noise Mapp. 2015 Jan;2(1):1-12.

30. De Coensel B, Sun K, Wei W, Van Renterghem T, Sineau M, Ribeiro C, et al. Dynamic Noise Mapping based on Fixed and Mobile Sound Measurements. In: Euronoise 2015, the 10th European Congress and Exposition on Noise Control Engineering. 2015.

31. Aumond P, Can A, De Coensel B, Botteldooren D, Ribeiro C, Lavandier C. Modeling Soundscape Pleasantness Using perceptual Assessments and Acoustic Measurements Along Paths in Urban Context. Acta Acust United Acust. 2017 May 1;103(3):430-43.

32. OpenStreetMap [Internet]. [cited 2017 May 30]. Available from: https://www.openstreetmap.org/\#map=16/49.6008/1.1135

33. geoR: Analysis of Geostatistical Data version 1.7-5.2 from CRAN [Internet]. [cited 2017 May 30]. Available from: https://rdrr.io/cran/geoR/

34. Cressie N. Statistics for Spatial Data. Revised Edition. Wiley; 2015. 928 p.

35. Diggle P, Ribeiro PJ. Model-based Geostatistics. Springer; 2007. 242 p.

36. West D. Introduction to Graph Theory - Second edition. 2nd ed. Prentice Hall; 2001.

37. Efron B. Bootstrap Methods: Another Look at the Jackknife. Ann Stat. 1979 Jan;7(1):1-26. 
38. Brocolini L, Lavandier C, Quoy M, Ribeiro C. Discrimination of urban soundscape through Kohonen map. In Edinburgh, Scotland; 2009 [cited 2017 Jul 26]. Available from: http://publietis.ensea.fr/2009/BLQR09/

39. Rey Gozalo G, Barrigón Morillas JM, Prieto Gajardo C. Urban noise functional stratification for estimating average annual sound level. J Acoust Soc Am. 2015 Jun 1;137(6):3198-208.

40. Barrigón Morillas JM, Escobar VG, Carmona JT, Sierra JAM, Vílchez-Gómez R, Río FJC del. Analysis of the prediction capacity of a categorization method for urban noise assessment. Appl Acoust. 2011 Oct $1 ; 72(10): 760-71$.

41. Bouttier F, Courtier P, Courtier P, Courtier P. Data Assimilation Concepts and Methods. 2002.

42. Tilloy A, Mallet V, Poulet D, Pesin C, Brocheton F. BLUE-based NO2 data assimilation at urban scale. J Geophys Res. 2013;4(118):2031-40.

43. Prieto Gajardo C, Barrigón Morillas JM, Rey Gozalo G, Vílchez-Gómez R. Can weekly noise levels of urban road traffic, as predominant noise source, estimate annual ones? J Acoust Soc Am. 2016 Nov;140(5):3702. 